\title{
Neuerungen durch Via sicura
}

\section{Geschäftsleitung und -führung des Fortbildungszentrums für Fahreignungsbegutachtung Schweiz}

Mit dem Teil der Via sicura, der am 1.7.2016 in Kraft tritt, sind Neuerungen vorgesehen, die die Ärzte direkt betreffen. Zwecks Qualitätssicherung müssen Ärzte, die Fahreignungsuntersuchungen durchführen, künftig gewisse Bedingungen erfüllen resp. Schulungen absolvieren. Der Schweizerischen Gesellschaft für Rechtsmedizin (SGRM) wurde die Aufgabe übertragen, die entsprechenden Schulungen durchzuführen.

\section{Einleitung}

Seit der Annahme des Verkehrssicherheitspakets Via sicura am 15.6.2012 das Parlament wurden verschiedene Massnahmen gestaffelt in Kraft gesetzt. Aus ärztlicher Sicht sind die Änderungen in Art. 15d des Strassenverkehrsgesetzes (SVG) die bisher wichtigsten.

Neben der Festsetzung der Tatbestände, die zu einer Fahreignungsabklärung bei Alkohol- und Drogendelikten führen, wurde die Entbindung vom Berufsgeheimnis für Ärzte* bei einer Meldung an die Behörde klar festgehalten. Ebenso wurde festgelegt, dass bei einer Meldung durch eine IV-Stelle über Vorliegen von Zweifeln an der Fahreignung eine Abklärung erfolgen muss [1].

Behörden dürfen Aufträge zu Fahreignungsabklärungen nur an entsprechend qualifizierte Ärzte erteilen.

Im Weiteren besteht seit 1.7.2014 die Vorschrift, dass bei Delikten, die Anlass zu Zweifeln an der Fahreignung geben, diese Untersuchungen nur durch entsprechend qualifizierte Ärzte und Psychologen durchgeführt werden dürfen, nämlich für verkehrsmedizinische $\mathrm{Ab}$ klärungen der Verkehrsmediziner SGRM und für verkehrspsychologische Abklärungen der Fachpsychologe für Verkehrspsychologie FSP [2].

Zum 1.7.2016 werden nun angepasste medizinische Mindestanforderungen in Kraft treten. Gleichzeitig werden im Sinne von Qualitätssicherungsmassnahmen auch die Bedingungen festgehalten, die Ärzte erfüllen müssen, die künftig Fahreignungsabklärungen durchführen. Somit sind sowohl die Ärzte wie auch deren Patienten direkt betroffen. Behörden dürfen Aufträge zu Fahreignungsabklärungen nur an entsprechend qualifizierte Ärzte erteilen.

\section{Medizinische Mindestanforderungen: Anhang 1 Verkehrszulassungsverordnung (VZV)}

In Zukunft wird es wie in der EU nur noch zwei medizinische Gruppen geben:

- Gruppe 1: Führerausweis-Kategorien A und B, Unterkategorien A1 und B1, Spezialkategorien F, G und M

- Gruppe 2: Führerausweis-Kategorien C und D, Unterkategorien $\mathrm{C} 1$ und D1, Bewilligung zum berufsmässigen Personentransport, Verkehrsexperten, Fahrlehrer

Die aktuell noch gültigen Mindestanforderungen aus den 1970er Jahren wurden überarbeitet. Teilweise werden die Mindestanforderungen auch nur punktuell angepasst. Beim Sehvermögen wird eine Annäherung an die EU-Richtlinien erfolgen und beim Diabetes mellitus werden detailliertere Regelungen festgehalten.

\section{Qualitätssicherung der Fahreignungs- beurteilung}

Die von Ärzten zu erfüllenden Voraussetzungen zur Durchführung von Fahreignungsabklärungen wurden gesetzlich festgelegt und insgesamt wurden vier verkehrsmedizinische Qualifikationsstufen definiert $[3,4]$. Grundsätzlich müssen die jeweiligen Ärzte über einen eidgenössischen oder anerkannten ausländischen Weiterbildungstitel verfügen.

Jede Stufe berechtigt zu bestimmten Untersuchungen, wobei die jeweils höheren Stufen auch die Berechtigung zur Durchführung von Untersuchungen der niedrigeren Stufen beinhalten [4]. Durch Festlegung der Voraussetzungen, die der Arzt für die jeweilige Berechtigung erfüllen muss, sollte schweizweit eine möglichst einheitliche Qualität und Beurteilung erzielt werden. 


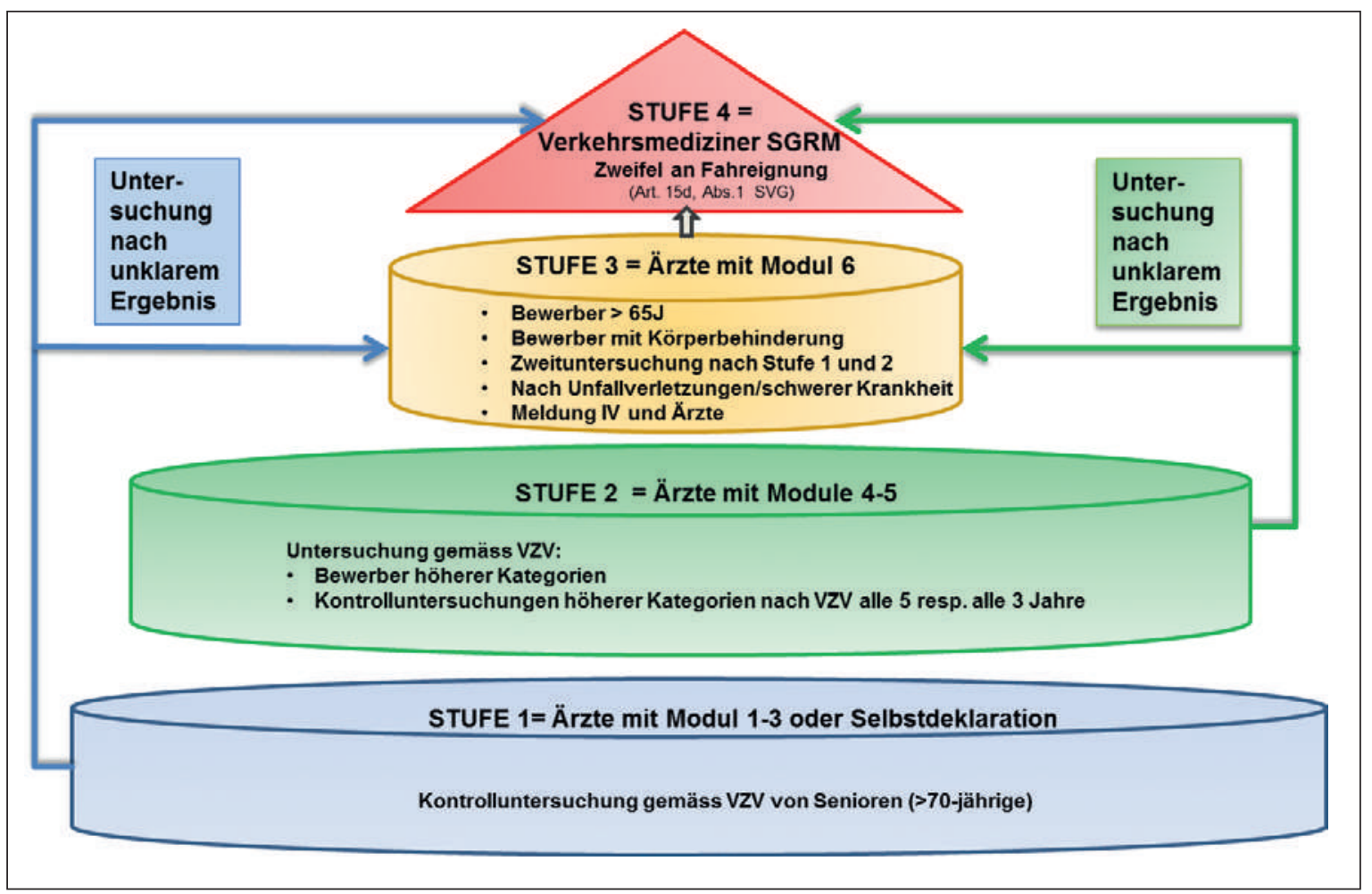

Abbildung 1: Das Stufenmodell.

Künftig darf jeder Arzt mit der jeweiligen Qualifikationsstufe die entsprechenden Untersuchungen durchführen und nicht wie bisher in einzelnen Kantonen nur vom Strassenverkehrsamt bestimmte Ärzte (z.B. Amts-, Bezirks- oder Vertrauensärzte).

Bei einem unklaren Ergebnis kann der Arzt dem Strassenverkehrsamt empfehlen, dass die Beurteilung durch eine Fachperson mit einer höheren Ausbildungsstufe vorgenommen wird (Abb. 1).

\section{Fortbildungszentrum für Fahreignungs- begutachtung Schweiz}

Die Organisation und Durchführung von Schulungen wurden vom Bundesamt für Strassen (ASTRA) der Schweizerischen Gesellschaft für Rechtsmedizin (SGRM) übertragen. Das in der SGRM organisatorisch integrierte Fortbildungszentrum für Fahreignungsbegutachtung Schweiz gewährleistet schweizweit eine einheitliche Schulung. Es werden Schulungen für alle Qualifikationsstufen angeboten. Die Kurse sind modular aufgebaut und vermitteln die notwendigen fachlichen Voraussetzungen, um die jeweiligen Untersuchungen nach den gängigen Qualitätsstandards durchzuführen.

Es wird für die Veranstaltung jeweils ein Antrag auf Crediterteilung bei der Fachgesellschaft SGIM/SGAM beantragt.

\section{Registrierung auf www.medtraffic.ch}

In Zusammenarbeit der SGRM mit der Vereinigung der Strassenverkehrsämter (asa) wurde eine InternetPlattform geschaffen (www.medtraffic.ch), auf der die wichtigsten Informationen bezüglich Fahreignungsuntersuchungen zu finden sind. Fragen können an info[at]medtraffic.ch gesendet werden.

Alle Ärzte, die Untersuchungen im Zusammenhang mit Fahreignung durchführen wollen, müssen sich auf dieser Homepage mit ihrer GLN (Global Location Number) und ihrem Geburtsdatum registrieren und die Administration der persönlichen Daten wird dann ebenfalls dort möglich sein. Das Geburtsdatum ist aus diesem Grund wichtig, weil der Gesetzgeber vorgesehen hat, dass die untersuchenden Ärzte nicht älter als 70 Jahre sein dürfen. Die Registrierung wird voraussichtlich ab November 2015 möglich sein. Zudem können Ärzte unter www.medtraffic.ch Kurse buchen und ihre Aus- und Fortbildung im Zusammenhang mit Fahreignungsabklärungen verwalten.

Auch die Strassenverkehrsämter haben Zugriff auf die für sie jeweils notwendigen Informationen und können bei Erhalt eines entsprechenden Zeugnisses, Berichtes oder Gutachtens die Qualifikationsstufe überprüfen. Zudem können die Fahrzeuglenker über diese Plattform Ärzte der benötigten Qualifikationsstufe suchen. 
Die Qualifikationsstufen sind schweizweit gültig und somit kann der Fahrzeuglenker über seinen Wohnkanton hinaus einen entsprechend qualifizierten Arzt aufsuchen.

\section{Verkehrsmedizinische Qualifikationsstufen $[3,4]$}

\section{Stufe 1}

Berechtigt zur Fahreignungsabklärung von Fahrzeuglenkern, die sich ab dem 70. Altersjahr alle 2 Jahre einer Untersuchung unterziehen müssen

Ärzte müssen über Kenntnisse und Fähigkeiten verfügen, die in Anhang $1^{\text {bis }}$ VZV festgelegt sind (ab 1.7.2016):

a. Kenntnis und Verständnis der für die verkehrsmedizinischen Kontrolluntersuchungen relevanten rechtlichen Grundlagen (SVG, SKV, VRV, VZV, kantonale Ausführungsbestimmungen);

b. Kenntnis der administrativen Abläufe zwischen der kantonalen Behörde und der untersuchenden Ärztin oder dem untersuchenden Arzt;

c. Kenntnis der Indikationen für verkehrsmedizinische Abklärungen, Zusatzuntersuchungen und ärztlich begleitete Kontrollfahrten sowie des diesbezüglichen Vorgehens;

d. Kenntnis des Untersuchungsgangs;

e. Fähigkeit zur Beurteilung der Fahreignung gemäss den medizinischen Mindestanforderungen in den einzelnen Diagnosegruppen sowie Erkennen eines Konsums problematischer Substanzen;

f. Kenntnis der verkehrsrelevanten Einschränkungen und Erkrankungen bei über 70-Jährigen und Fähigkeit, die Fahreignung, insbesondere bei Vorliegen von kognitiven Defiziten, zu beurteilen;

g. Kenntnis der verschiedenen medizinischen Richtlinien der Fachgesellschaften (z.B. Richtlinien bezüglich Fahreignung bei Diabetes mellitus der Schweizerischen Gesellschaft für Endokrinologie und Diabetologie) und Fähigkeit, diese anzuwenden;

h. Kenntnis der Auflagen, welche die kantonale Behörde verfügen kann;

i. Fähigkeit, die Informationen richtig den kantonalen Behörden zu übermitteln (Anhang $3 \mathrm{VZV}$ ).

Ärzte können selbst entscheiden, wie sie sich dieses Knowhow aneignen. Sie können mittels Selbstdeklaration unter www.medtraffic.ch bestätigen, dass sie über die notwendigen Kenntnisse und Fähigkeiten verfügen.

Die Fahreignungsuntersuchung an und für sich stellt für den Hausarzt in der Regel kein Problem dar. Erfahrungsgemäss kommt es jedoch bei der Beurteilung der Fahreignung im gesetzlichen Kontext häufig zu Fehleinschätzungen, da teilweise die gesetzlichen Voraussetzungen unbekannt sind, diese unterschiedlich interpretiert werden, dort, wo Auflagen zu empfehlen wären, keine formuliert werden oder bei der Formulierung von Auflagen solche empfohlen werden, die von den Behörden nicht umgesetzt werden können.

Von der SGRM wird ein eintägiger Kurs angeboten (Modul 1-3). Zentrales Thema hier ist die Vermittlung der gesetzlich vorgeschriebenen Kenntnisse. Mit dem Kurs wird ein Handout abgegeben, das bei der Fahreignungsbeurteilung beigezogen werden kann.

Obwohl in der gesetzlichen Vorgabe kein Muss für die Absolvierung eines derartigen Kurses besteht, wird durch diese eintägige Fortbildung ein umfassendes Wissen erworben, das auch bei allfälligen juristischen Auseinandersetzungen mit der untersuchten Person oder mit den Behörden hilfreich sein kann, zumal juristische Kenntnisse und das forensische Denken in der alltäglichen Praxis nicht von zentraler Bedeutung sind, aber im Zusammenhang mit der Rolle des Arztes als Sachverständiger bei der Fahreignungsabklärung eine entscheidende Rolle spielen.

\section{Stufe 2}

Berechtigt zur Fahreignungsabklärung von Inhabern höherer Führerausweiskategorien und von Bewerbern um höhere Führerausweiskategorien.

Für die Stufe 2 ist ein eintägiger Kurs obligatorisch (Modul 4-5). Der vorgängige Besuch der Module 1-3 wird von der SGRM dringend empfohlen, falls ein Arzt eine höhere Qualifikationsstufe erreichen möchte, denn damit gehen auch höhere Anforderungen an den untersuchenden Arzt einher, was einer soliden Grundausbildung bedarf.

\section{Stufe 3}

Berechtigt Ärzte, einfache Gutachten zu erstellen, Untersuchungen bei Personen nach unklarem Ausgang bei einer Erstabklärung bei einem Arzt der Stufe 1 oder 2 durchzuführen und Fälle nach Art. 15 Abs. 1 lit. d und e abzuklären (z.B. körperbehinderte Personen, Meldung durch IV-Stelle). Stufe 1 und 2 sind Grundvoraussetzung. Die Ausbildung zur Stufe 3 besteht aus einem zusätzlichen Kurs (Modul 6A) und 5 Gutachten unter Supervision eines Verkehrsmediziners SGRM (Modul 6B).

\section{Stufe 4 = Verkehrsmediziner SGRM}

Berechtigt Ärztinnen und Ärzte alle in Frage kommenden Untersuchungen zur Fahreignung durchzuführen. Voraussetzung ist die Ausbildung zum Verkehrsmediziner SGRM [5]. 


\section{Anerkennung der verkehrsmedizinischen Qualifikation}

Die Anerkennung erfolgt durch die kantonale Behörde, in der der Arzt vorwiegend tätig ist. Sie gilt für die ganze Schweiz und ist 5 Jahre, maximal bis zum 70. Altersjahr, gültig [6-8].

\section{Verlängerung der Anerkennung}

Ärzte der Stufe 1 müssen der kantonalen Behörde alle fünf Jahre bestätigen, dass ihr Wissen auf dem neusten Stand ist. Um ihr Wissen à jour zu halten, müssen Ärzte der Stufen 2 und 3 alle fünf Jahre eine vierstündige Fortbildung (Refresher) besuchen. Verkehrsmediziner SGRM müssen die Bedingungen zur Beibehaltung ihres Titels gemäss Titelreglement erfüllen [9].

\section{Übergangsregelung}

In einigen Kantonen haben bereits ab 2010 durch die SGRM organisierte Schulungen stattgefunden. Die Ärzte, die bereits Kurse absolviert haben und damit verbunden auch Qualifikationsstufen durch die kantonale Behörde erhalten haben, behalten nach wie vor

\section{Durch die eintägige Fortbildung wird ein umfassendes Wissen erworben.}

ihre Qualifikationsstufe. Diese ist dann bis zum 30.6.2021 gültig und erst danach benötigt es eine obligatorische Fortbildung. Das heiss die Gültigkeit beginnt erst mit Inkrafttreten der neuen Gesetzgebung auf 1.7.2016.

Korrespondenz:

SGRM

Fortbildungszentrum für

Fahreignungsbegutachtung

Schweiz der Schweize-

rischen Gesellschaft

für Rechtsmedizin

Winterthurerstrasse 190

$\mathrm{CH}-8057$ Zürich

info[at]medtraffic.ch
Vom Gesetzgeber sind auch weitere Übergangfristen vorgesehen [10]:

- Bis 31.12.2017 dürfen Ärzte der Stufe 1 die Untersuchung der über 70-jährigen Fahrzeuglenker alle 2 Jahre weiterhin nach bisherigem Recht ohne Anerkennung der kantonalen Behörde durchführen.

- Medizinische und verkehrspsychologische Gutach- ten sind nach bisherigem Recht in allen Kantonen bis zum 31.12.2018 anzuerkennen, wenn sie von einer von der kantonalen Behörde bezeichneten Untersuchungsstelle verfasst und nicht älter als ein Jahr sind.

- Die Module 4-6 der verkehrsmedizinischen Fortbildung der SGRM, die ab dem 1.7.2010 besucht wurden, werden bei der Anerkennung berücksichtigt.

- Die kantonalen Behörden können verkehrsmedizinische Untersuchungen, die von einem Arzt Stufe 4 durchgeführt werden müssen, bis zum 31.12.2019 auch durch Personen ohne entsprechende Anerkennung durchführen lassen oder entsprechende Untersuchungsergebnisse anerkennen, wenn die Person auch bisher entsprechende Untersuchungen durchgeführt hat und die zu untersuchende Person wegen personellen Kapazitätsengpässen bei Ärzten mit einer entsprechenden Anerkennung unverhältnismässig lange auf die Durchführung der Untersuchung warten müsste (sog. «Ventilklausel»).

Nach den Übergangsfristen gilt dann definitiv die neue Gesetzgebung. Im Zweifelsfall muss der jeweilige Arzt mit der zuständigen kantonalen Behörde Kontakt aufnehmen.

Danksagung
Wir möchten uns für die gute und konstruktive Zusammenarbeit be
der asa bedanken.
Referenzen
$\left.\begin{array}{ll}1 & \text { Art. 15d SVG } \\ 2 & \text { Art. 28a SVG } \\ 3 & \text { www.news.admin.ch/NSBSubscriber/message/attachments/ } \\ & 40154 . p d f \\ 4 & \text { Art. 5a }{ }^{\text {bis }} \text { VZV (ab 1.7.2016 gültig) } \\ 5 & \text { www.sgrm.ch/uploads/media/VM_Titelreglement_2013_- } \\ & \text { genehmigt_d.pdf } \\ 6 & \text { Art. 5d VZV } \\ 7 & \text { Art. 5e VZV } \\ 8 & \text { Art. 5g VZV } \\ 9 & \text { Art. 5f VZV } \\ 10 & \text { Art. } 151 j \text { VZV }\end{array}\right\}$ (jeweils ab 1.7.2016 gültig)

